\title{
Optimization of Injection Process Parameters to Reduce Warpage in Glass Fiber-Reinforced Polyamide Molded Parts
}

\author{
Jian Faping ${ }^{\mathrm{a}}$, Du Jitao ${ }^{\mathrm{b}}$, Yang $\mathrm{Mei}^{\mathrm{c}}$ \\ Advanced Technical Vocational Institute, Shanghai University of Engineering Science, Shanghai \\ 200437, China \\ aemail:13816828620@139.com, bemail:djtjaster@126.com
}

Keywords: sharp corner effect; differential shrinkage; warpage deformation; parameter optimization; glass fiber orientation

\begin{abstract}
The warpage in a glass fiber-reinforced polyamide molded part caused by the sharp corner effect and differential shrinkage could be effectively reduced by optimizing the injection process parameters using numerical simulations. The results showed that adopting a three-gate injection method could effectively minimize the warpage caused by the sharp corner effect by more than $66 \%$ due to better fiber orientation and compensation of the shrinkage deviations. Simple equations were used to obtain the optimized packing profile, and the overall warpage could be reduced by more than $43 \%$.
\end{abstract}

\section{Introduction}

Warpage is one of the most common defects in injection-molded parts, and glass fiber (GF)-reinforced polyamide (PA) molded parts are no exception. For several years, scientists have tried to reduce warpage defects that occur during the fabrication process using computer aided engineering (CAE) and design of experiments (DOE). For instance, Chen W. C. et al. have successfully explored the application of the Taguchi method to improve the fabrication process ${ }^{[1]}$. Xia Wei et al. proposed the use of a Gaussian process surrogate model combined with DOE to minimize the warpage of injection-molded parts ${ }^{[2]}$. However, they only focused on the model and algorithm. Aihua Xiong et al. and Hu Yan et al. studied the impact of glass fiber-reinforcement on the warpage, but the warpage caused by the sharp-corner effect has not been considered so far ${ }^{[3,4]}$.

In this study, the warpage of an electric switch support was minimized by reducing the warpage caused by both the sharp-corner effect and differential shrinkage. Furthermore, the fiber orientation at the sharp corner and the pressure curve optimization are also discussed in this paper.

\section{Development of numerical simulation model}

The average wall thickness of the electric switch support was $3 \mathrm{~mm}$, and the overall dimensions of the three-dimensional model shown in Fig. 1 are $48 \mathrm{~mm} \times 63 \mathrm{~mm} \times 39 \mathrm{~mm}$. Although not a thin-wall part, the groove and clips on its top, shown in Fig. 1, are key assembly positions and the accuracy required (small size deviation) is relatively high, and therefore the warping must be strictly controlled. Midplane, 3D and Fusion are the three major mesh types available in the Moldflow software ${ }^{[5]}$. The surface meshes (Fusion mesh type) were designed as shown in Fig. 2, and the grid mesh tool was used to modify the mesh defects. Finally, the mesh that satisfied the requirements for the subsequent analysis consisted of 10106 grids and 5037 nodes, which resulted in a match percentage of $91.3 \%$. 


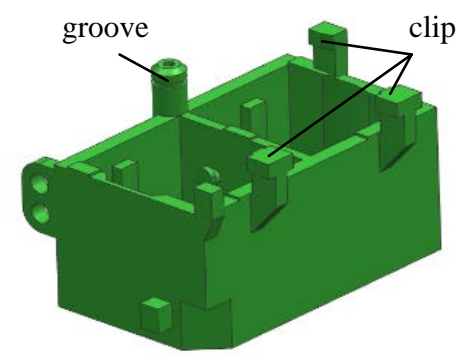

Fig. 1. 3D model of molded part.

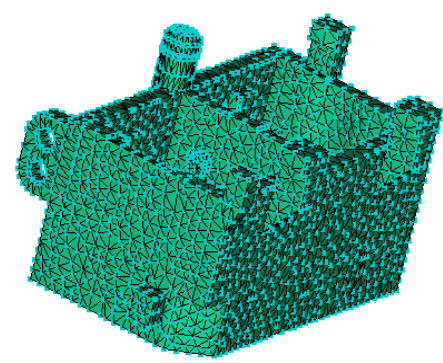

Fig. 2. Mesh model of molded part.

\section{Initial CAE simulation}

In this study, PA reinforced with 30 vol. \% GF, denoted as PA66+30\%GF (Manufacturer: DuPont Engineering Polymers, Designation: Zytel 101L (30\%EGF)), was used as the raw material, and it was manufactured as described in the literature [4]. The initial simulation was carried out using this material and the default Moldflow parameters, which resulted in a warpage of $0.4165 \mathrm{~mm}$. The warpages caused by differential cooling, differential shrinkage, orientation effect and sharp corner effect were $0.0221,0.2160,0.1379$ and $0.3220 \mathrm{~mm}$, respectively. The warpage could mainly be attributed to the sharp corner effect (46.132\%) and differential shrinkage (30.946\%). Therefore, reducing the warpage caused by the sharp corner effect and differential shrinkage should result in a significant overall reduction of the warpage.

\section{Warpage reduction}

\section{Reduction of warpage caused by sharp corner effect}

Optimizing the gate location and number of gates should lead to a significant reduction in the warpage caused by the sharp corner effect, because multi-gate injection was demonstrated to significantly improve the single orientation of the GF and thereby reduce the residual stress on the flowing material, which is one of the main factors that causes warpage ${ }^{[6]}$. Utilizing CAE, the best gate location could be obtained quickly and accurately. In Fig. 3, the deep-blue areas represent the best gate locations when using one gate, two gates, three gates or four gates.

Fig. 4 shows the warpage that occurred for the different amounts of gates. It can be seen that a multi-gate injection could effectively reduce the warpage caused by the sharp corner effect. When using three gates for injection, the warpage caused by the sharp corner effect was $0.1082 \mathrm{~mm}$, which was a reduction of $66.398 \%$ compared to the $0.3220 \mathrm{~mm}$ obtained when using only one gate for injection. Apparently, adopting a three-gate injection method changed the melt flow direction and the glass fiber orientation, which resulted in the best possible warpage balance in three dimensional space ${ }^{[7]}$. Fig. 5 reveals the optimal fiber orientation in three-dimensional space $\left(\theta \leqslant 45^{\circ}\right)$. The average fiber orientations at the sharp corner for the different amounts of gates are shown in Fig. 6. Glass fiber-reinforced PA is an anisotropic material, and shrinkage is different in each direction. However, the shrinkage at the sharp corner can be compensated if the fiber orientation in each direction is similar, as illustrated in Fig. 5. The fiber orientation in the sharp corner obtained for a three-gate injection process , as shown in Fig. 6(c), best matched the optimal orientation, and the fiber distribution was more uniform, thereby effectively reducing warpage. 


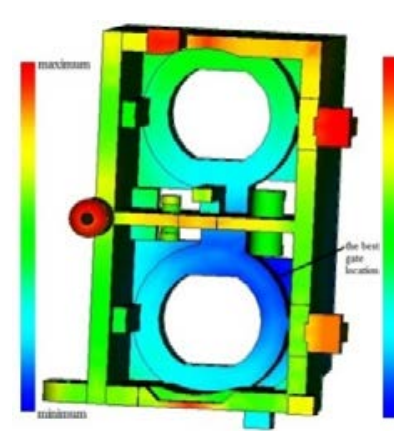

(a) One gate

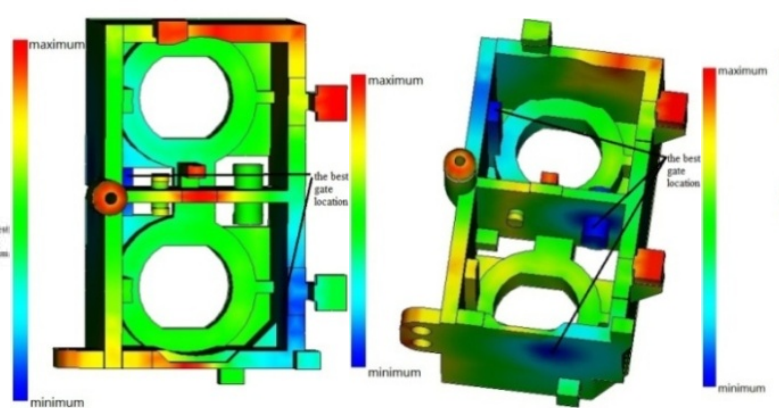

(b) Two gates (c) Three gates

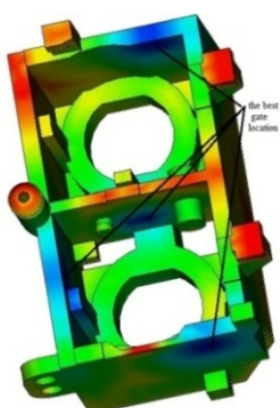

(d) Four gates

Fig. 3. Best gate locations obtained by CAE simulations.

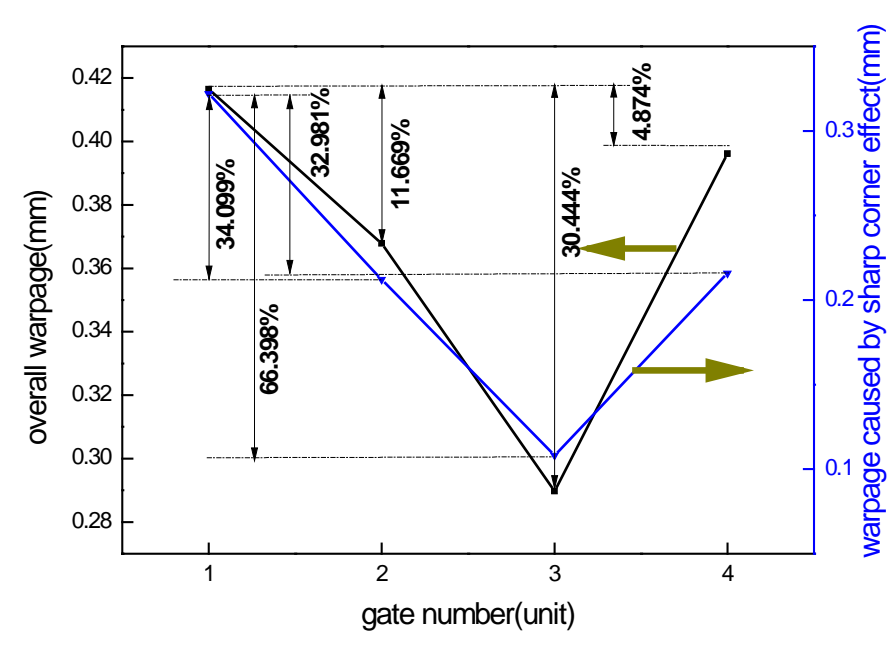

Fig. 4. Warpage obtained for different amounts of gates.

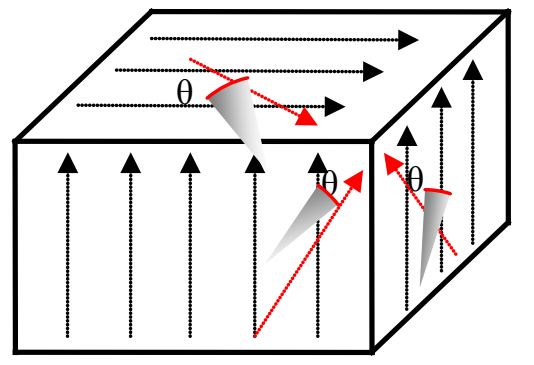

Fig. 5. Optimal fiber orientation model.

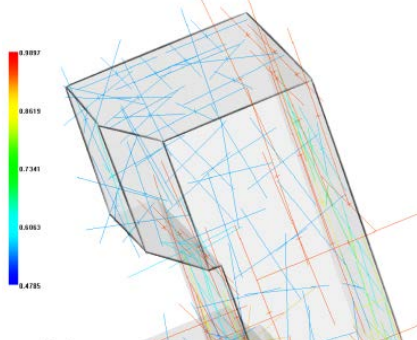

(a) One gate

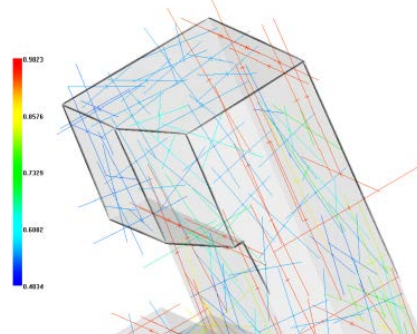

(b) Two gates

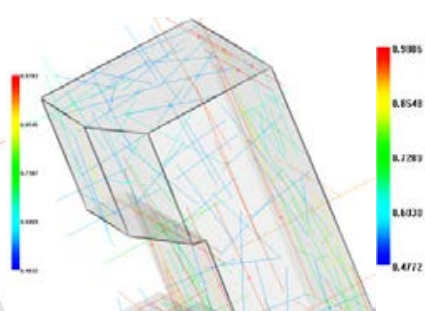

(c) Three gates

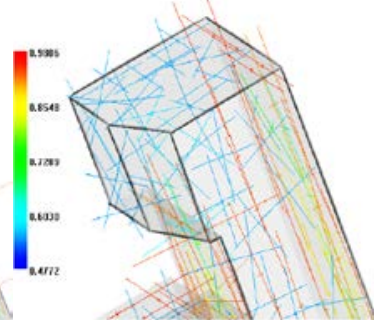

(d) Four gates

Fig. 6. Fiber orientation in sharp corner for different amounts of injection gates.

\section{Reduction of warpage caused by differential shrinkage}

According to experience and the simulation results, the main factors affecting the warpage caused by differential shrinkage were melt temperature, mold temperature, injection time, packing curve and cooling time.

\section{Optimization of holding pressure curve}

The packing process is a very important step during injection molding, and the pressure setting directly determines the injection quality. The optimized holding pressure curve was obtained with Moldflow $^{[8]}$. Typically, the holding pressure remains constant at first and then decays. The initial packing curve required that $80 \%$ of the injection pressure be retained for $30 \mathrm{~s}$, and the gate freeze time was $28.22468 \mathrm{~s}$, which indicated that a holding time of $30 \mathrm{~s}$ was reasonable. The volume shrinkage of the filling extremity after ejection was $4.109 \%$, which exceeded the volume shrinkage 
requirements for PA66+30\%GF parts. Therefore, the holding pressure had to be increased. When the holding pressure was changed to $120 \%$ of the injection pressure, the requirements of $3 \%$ volume shrinkage could be met. The optimal packing curve was obtained using the following simple equations:

$$
\begin{gathered}
T c=\frac{T m+T z}{2}-T p \\
T d=T f-T c-T p
\end{gathered}
$$

where $T_{c}$ denotes the holding time of the constant pressure stage (in s), $T_{m}$ is the time when the pressure of the filling extremity node reaches its maximum value (in $\mathrm{s}$ ), $T_{z}$ is the time when the pressure of the filling extremity node reaches zero (in s), $T_{p}$ is the moment when V/P is switched (in s), $T_{d}$ refers to the holding time of the decaying pressure stage (in s) and $T_{f}$ is the time when the frozen layer fraction of the gate node is equal to 1 (in s).

In this experiment, $T_{m}=21.97428 \mathrm{~s}, T_{z}=4.97428 \mathrm{~s}, T_{p}=2.3328 \mathrm{~s}$ and $T_{f}=28.22468 \mathrm{~s}$. According to the literature, ${ }^{[7]}$ the packing curve should obey the second-order decay law, and a moment when the filling extremity is in a low pressure state is a good starting point to initiate the second-order decay. In this study, the holding pressure started to rapidly decrease to $30 \%$ of the injection pressure at 11.14148 s, i.e., it rapidly dropped to $30 \%$ of the injection pressure within $3.593 \mathrm{~s}$, and then slowly decreased to zero within another $12.91 \mathrm{~s}$. The final packing curve is shown in Fig. 7.

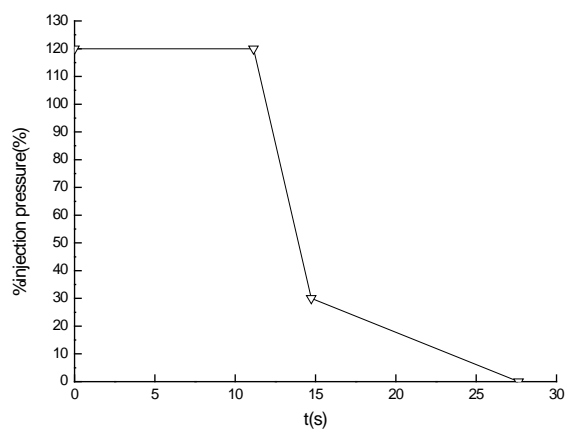

Fig. 7. Final packing curve.

\section{Optimization of other process parameters}

In the following tables, the letters $\mathrm{A}\left(315^{\circ} \mathrm{C}, 325^{\circ} \mathrm{C}, 335^{\circ} \mathrm{C}, 345^{\circ} \mathrm{C}\right), \mathrm{B}\left(25^{\circ} \mathrm{C}, 35^{\circ} \mathrm{C}, 45^{\circ} \mathrm{C}, 55^{\circ} \mathrm{C}\right)$, C $(0.5 \mathrm{~s}, 1 \mathrm{~s}, 1.5 \mathrm{~s}, 2 \mathrm{~s})$ and $\mathrm{D}(15 \mathrm{~s}, 20 \mathrm{~s}, 25 \mathrm{~s}, 30 \mathrm{~s})$ denote the melt temperature, mold temperature, injection time and cooling time, respectively. Then, the orthogonal testing method was adopted and the results of the CAE simulations are compared in Table 1.

Table 1. Results of orthogonal experiments.

\begin{tabular}{ccccccc}
\hline No. & $\mathrm{A}\left({ }^{\circ} \mathrm{C}\right)$ & $\mathrm{B}\left({ }^{\circ} \mathrm{C}\right)$ & $\mathrm{C}(\mathrm{s})$ & $\mathrm{D}$ & Warpage & S/N value \\
\hline 1 & 315 & 25 & 0.5 & 15 & 0.2576 & -11.7811 \\
\hline 2 & 315 & 35 & 1 & 20 & 0.2791 & -11.0848 \\
\hline 3 & 315 & 45 & 1.5 & 25 & 0.2693 & -11.3953 \\
\hline 4 & 315 & 55 & 2 & 30 & 0.2821 & -10.9920 \\
\hline 5 & 325 & 25 & 1 & 25 & 0.2610 & -11.6672 \\
\hline 6 & 325 & 35 & 0.5 & 30 & 0.2459 & -12.1848 \\
\hline 7 & 325 & 45 & 2 & 15 & 0.2864 & -10.8605 \\
\hline 8 & 325 & 55 & 1.5 & 20 & 0.2740 & -11.2450 \\
\hline 9 & 335 & 25 & 1.5 & 30 & 0.2611 & -11.6639 \\
\hline 10 & 335 & 35 & 2 & 25 & 0.2702 & -11.3663 \\
\hline
\end{tabular}




\begin{tabular}{ccccccc}
\hline 11 & 335 & 45 & 0.5 & 20 & 0.2434 & -12.2736 \\
\hline 12 & 335 & 55 & 1 & 15 & 0.2751 & -11.2102 \\
\hline 13 & 345 & 25 & 2 & 20 & 0.2609 & -11.6705 \\
\hline 14 & 345 & 35 & 1.5 & 15 & 0.2646 & -11.5482 \\
\hline 15 & 345 & 45 & 1 & 30 & 0.2508 & -12.0134 \\
\hline 16 & 345 & 55 & 0.5 & 25 & 0.2561 & -11.8318 \\
\hline
\end{tabular}

Table 2. Analysis of effect of process parameters on warpage.

\begin{tabular}{ccccc}
\hline $\mathrm{K}$ & $\mathrm{A}$ & $\mathrm{B}$ & $\mathrm{C}$ & $\mathrm{D}$ \\
\hline $\mathrm{K}_{1}$ & -11.3133 & -11.6957 & -12.0178 & -11.3500 \\
\hline $\mathrm{K}_{2}$ & -11.4894 & -11.5460 & -11.4939 & -11.5685 \\
\hline $\mathrm{K}_{3}$ & -11.6285 & -11.6357 & -11.4631 & -11.5652 \\
\hline $\mathrm{K}_{4}$ & -11.7660 & -11.3200 & -11.2223 & -11.7135 \\
\hline $\mathrm{R}$ & 0.4527 & 0.3757 & 0.7955 & 0.3635 \\
\hline Sequence & 2 & 3 & 1 & 4 \\
\hline
\end{tabular}

The results of the orthogonal tests are shown in Table 2. To identify the impact of the different molding process parameters, the rank was used to analyze the results of the orthogonal experiments. As demonstrated by the $R$ values in Table 2, when ranked according to their impact on the warpage deformation, the following sequence of the process parameters was obtained (highest to lowest): injection time, melt temperature, mold temperature and cooling time. Considering the results in Table 2 and the process requirements, the optimized parameter combination is $\mathrm{A}_{4} \mathrm{~B}_{1} \mathrm{C}_{1} \mathrm{D}_{4}$, which is melt temperature $345^{\circ} \mathrm{C}$, mold temperature $25^{\circ} \mathrm{C}$, injection time $0.5 \mathrm{~s}$ and cooling time 30s. Using the optimized process parameters, the warpage obtained with Moldflow was $0.2354 \mathrm{~mm}$ (cp. Fig. 8), which corresponds to a reduction of $43.481 \%$ compared to the initial simulation.

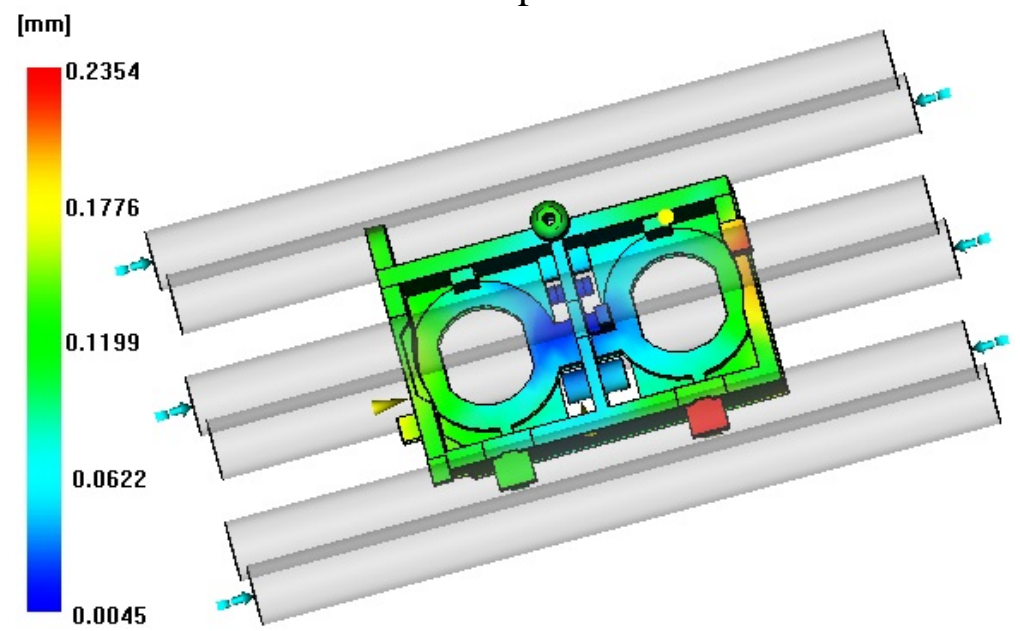

Fig. 8. Optimized warpage.

\section{Conclusions}

The warpage deformation of a GF-reinforced PA injection-molded part was reduced by modifying the injection process parameters and the following conclusions were obtained: (1) using a three-gate injection process could effectively reduce the warpage caused by the sharp corner effect by $66.389 \%$, which provided a reference for further reduction of the warpage caused by the sharp corner effect, and (2) the optimized holding pressure curve was obtained using the equations presented in this paper, which considered both the pressure at the filling extremity and the frozen layer fraction at the gate node. The results are expected to provide a theoretical basis for the production of qualified PA components. 


\section{Acknowledgment}

This project was supported by the Scientific Research Fund of the Advanced Vocational Technical College of the Shanghai University of Engineering Science (Grant No. A-2501-11-0015).

\section{References:}

[1] W.C. Chen, M.W. Wang, G.L. Fu et al. Optimization of plastic injection molding process via Taguchi's parameter design method, BPNN, and DFP. Proceedings of the Seventh International Conference on Machine Learning and Cybernetics,vol. 6, pp.3315-3321, July 2008.

[2] Wei Xia, Bin Luo, Xiaoping Liao. An enhanced global optimization method based on Gaussian process and its application of warpage control in injection molding. Proceedings of the 2010 IEEE International Conference on Information and Automation, pp. 970-975. June 2010.

[3] Aihua Xiong, Hesheng Liu, Xingyuan Huang et al. Influence of processing parameters on injection pressure and warpage of short-glass fiber reinforced PP injection-molded part. Polymer Materials Science and Engineering, vol.28, No.6, pp.163-168, June 2012.

[4] $\mathrm{Hu}$ Yan and Li Jiquan, Impact of glass fiber on warpage in injection-molded parts. Light Industry Machinery, vol.30, No.3, pp.13-16.June 2012.

[5] M.J.Wang, and L.F.Tang, Optimal design to gate-location based on Moldflow, Journal of Anhui University of Technology and Science, vol. 23, pp.12-15, December 2008.

[6] Zuo Daping, Zhang Yihua, Rui Yulong. Study on warpage of PA66/GF part. Engineering Plastic Application, vol. 33, No.12, pp.23-25, 2005.

[7] Aihua Xiong, Hesheng Liu, Xingyuan Huang et al. Simulated study on effect of fiber parameters on fiber orientation and injection-molded part's deflection. Materials Science \& Technology, vol. 20, No.3, pp.132-138, June 2012.

[8] Deqiang Zhang, Yan Zhang, Xiaoxin Meng et al. Packing curve optimization methods based on Moldflow. Journal of Liaoning University of Technology (Natural Science Edition), Vol.35, No.1, pp. 37- 40, Feb.2015. 